\title{
Soixante-neuf ans après avoir survécu à l'épidémie de polio
}

\author{
Barry J. McMahon, Sue Dojeiji MD MÉd, Rene A. Leiva MD, Joan M. Fisher BSc inf. MÉd
}

— Citation : CMAJ 2021 December 20;193:E1915-7. doi : 10.1503/cmaj.210985-f

Voir la version anglaise de l'article ici : www.cmaj.ca/lookup/doi/10.1503/cmaj.210985

A ujourd'hui âgé de 72 ans, Barry McMahon, producteur de films et de vidéos à la retraite, a reçu un diagnostic de poliomyélite paralytique aiguë en 1951, alors qu'il avait 3 ans. Ses muscles respiratoires et les muscles de ses bras et de ses jambes ont été affectés, mais il n'a pas eu besoin de poumon d'acier. Il a passé des mois en isolement à l'hôpital, suivis de plusieurs mois de convalescence et de réadaptation. Son premier signe de récupération a été la mobilité gagnée au niveau de son orteil gauche.

Barry a toujours sa faiblesse aux bras et aux jambes, particulièrement du côté droit. Il a appris à marcher sans dispositif d'aide à la déambulation, mais il a eu besoin d'une orthèse genou-chevillepied pour soutenir sa jambe droite; il l'a utilisée pendant 8 ans. Il a subi une arthrodèse et des transferts de tendons à la cheville droite pour soutenir son pied et sa cheville droits et avoir une démarche sécuritaire. Cette faiblesse ne s'est jamais améliorée, mais il a appris à vivre avec elle. Il a été en mesure de travailler, d'élever une famille et de réaliser différents projets.

Dans les années 1980, Barry s'est fracturé le fémur en faisant une vilaine chute. En 1990, il a commencé à utiliser un fauteuil roulant électrique pour éviter les chutes et composer avec la baisse de son endurance musculaire.

En 1996, sans avoir subi de traumatisme ou été atteint d'une maladie spécifique, Barry a soudainement développé un surcroît de fatigue, vu son endurance musculaire diminuer et commencé à ressentir une faiblesse accrue et des douleurs généralisées et réfractaires. Compte tenu de ses antécédents de polio et de l'absence d'autres causes identifiables, un physiatre a diagnostiqué un syndrome postpolio ${ }^{1}$. Toujours en 1996, comme ses symptômes persistaient, Barry a présenté une demande de prestations d'invalidité de longue durée. Malgré ses difficultés, il a continué de faire du bénévolat auprès d'organismes de bienfaisance communautaires.

Plusieurs facteurs font que les symptômes de syndrome postpolio de Barry sont difficiles à gérer. L'un des défis consiste à trouver des professionnels de la santé qui connaissent bien les effets à long terme de la polio et le traitement à adopter pour ne pas déclencher de nouveaux symptômes. Le symptôme le plus difficile à maîtriser est la douleur. Celle de Barry est multifactorielle; elle est constante, généralisée et d'intensité variable selon l'activité, ce qui est fréquent avec le syndrome postpolio.

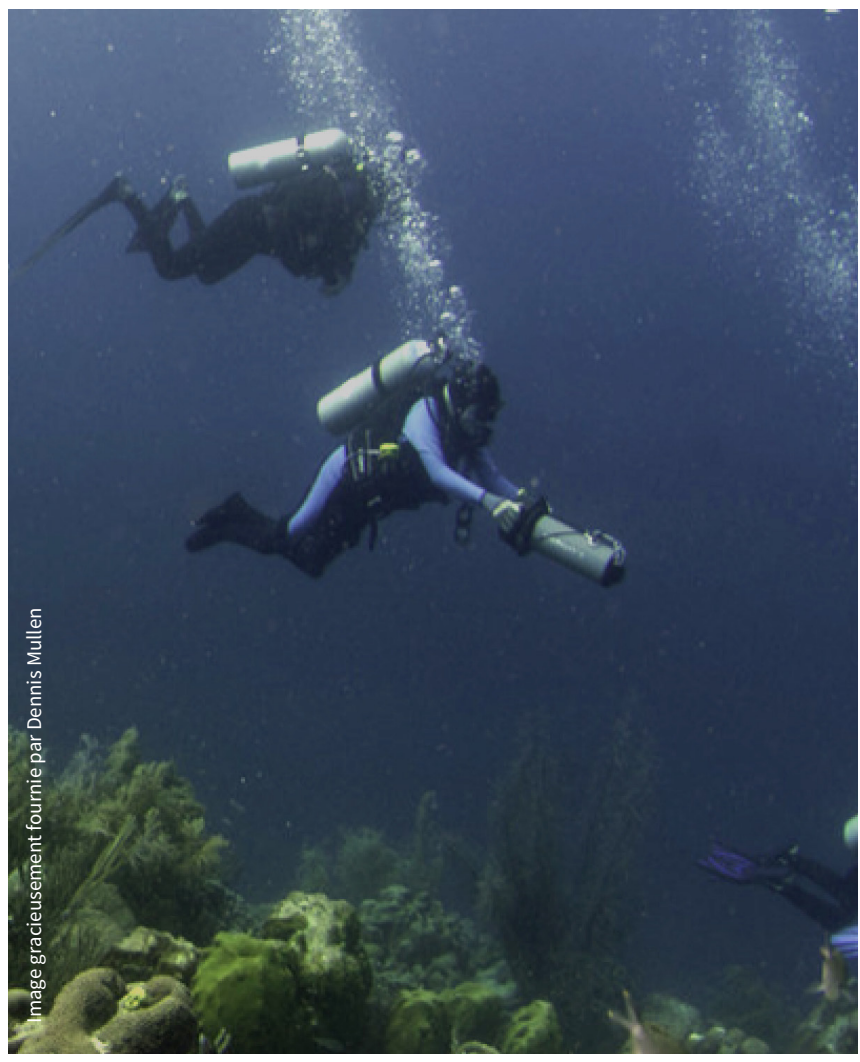

Barry McMahon en action; il utilise un propulseur de plongée.

\section{Point de vue du patient}

On estime à 31000 le nombre de survivants de la polio au Canada, et moins de la moitié d'entre nous présenteraient des symptômes de syndrome postpolio. Nous ne formons pas une grosse cohorte, alors nous faisons face à l'inconnu en matière de soins de santé.

J'ai utilisé plusieurs dispositifs d'aide (ils évoluent sans cesse), et mon domicile respecte les principes d'accessibilité universelle ${ }^{2}$, mais les interventions dont j'ai le plus bénéficié sont l'aide de mon âme sœur, de mes filles et des préposées que m'envoie mon centre communautaire. 
À part quelques petites études réalisées dans les dernières décennies ${ }^{3}$, peu de recherches cliniques ont porté sur le syndrome postpolio. Je présume que c'est dû au fait que notre cohorte est peu nombreuse, que d'année en année, ses membres décèdent et que l'industrie pharmaceutique n'y trouve pas son compte.

Donc, faute de données tirées d'études cliniques, les médecins font de leur mieux; ils utilisent leur jugement et mettent à profit l'expérience qu'ils acquièrent en traitant des personnes atteintes de polio et d'autres maladies. On s'ajuste au fur et à mesure. Par exemple, on m'a prescrit du méthylphénidate pour ma fatigue. Après quelques années, je l'ai cessé parce que j'ai commencé à douter de son effet sur mes symptômes de fatigue et de douleur. En lisant sur ce produit, j'ai découvert qu'il exerçait des bienfaits limités chez certaines personnes atteintes du syndrome de fatigue chronique ${ }^{4}$, mais je n'ai trouvé aucune donnée sur son efficacité pour le syndrome postpolio.

Il semble que la polio affecte chaque survivant différemment; c'est pourquoi la prise en charge des symptômes doit être individualisée. Les forums en ligne à l'intention des survivants de la polio s'accordent pour dire qu'on en sait réellement peu, même s'il existe plusieurs théories. Étant donné que ce qui compte le plus, c'est la qualité de vie, les discussions en ligne permettent d'aborder beaucoup de suggestions pour la prise en charge des symptômes, mais on voit clairement que les survivants de la polio ont besoin des conseils éclairés de leurs médecins.

Il y a environ 14 ans, j'ai eu la chance de découvrir que je pouvais faire de la plongée avec l'aide d'un instructeur et d'une équipe spécialisés pour les personnes handicapées. L’hiver, je fais de la plongée autour des récifs de l'île Bonaire dans les Antilles néerlandaises. L'été, les épaves qui jonchent le fond du fleuve Saint-Laurent nous en mettent plein la vue. Après plus de 260 plongées, je suis convaincu d'en avoir tiré des avantages thérapeutiques et j'espère continuer le plus longtemps possible.

En général, les survivants de la polio sont des gens très indépendants ${ }^{5}$; nous sommes habituellement tout à fait capables de nous y retrouver lorsqu'il est question de santé. Ceux d'entre nous qui ont d'importantes limites sur le plan de la mobilité apprécient la télémédecine, qui gagne en popularité (en partie grâce à la pandémie), surtout l'hiver. Je me sens plus proche de mon équipe soignante et j'espère que cette tendance se maintiendra. Cela dit, les rendez-vous en personne sont bien sûr encore nécessaires pour les examens physiques et les chirurgies.

Plusieurs éléments de mon syndrome postpolio demeurent mystérieux. Comment savoir si mes autres symptômes (intolérance au froid, sensibilité aux médicaments, problèmes de déglutition, gain pondéral et œdème) font partie du syndrome postpolio ou s'ils sont plutôt dus à d'autres causes sous-jacentes? Quels sont les effets du syndrome postpolio sur le processus normal de vieillissement?

J'ai toujours cru que l'objectif est de maintenir un équilibre entre la santé physique, la santé mentale et la santé spirituelle. Lorsque cet équilibre est atteint, ma résilience augmente face aux pressions qui changent constamment. - Barry J. McMahon

\section{Point de vue d'une amie proche}

Barry et moi nous sommes rencontrés il y a 4 ans; nous avions respectivement perdu nos conjoints 4 ans et 3 ans auparavant.
Je savais ce qu'était la polio : lorsque j'étais enfant, les écoles de Terre-Neuve avaient fermé durant l'épidémie de 1959. Mon premier emploi, en 1968, était dans un centre de réadaptation pour enfants à St. John's, où certains patients apprenaient à composer avec les effets d'une polio contractée 9 ans plus tôt.

Je n'avais par contre jamais entendu parler du syndrome postpolio avant de rencontrer Barry. Barry est extrêmement indépendant. J'ai été impressionnée de voir que même confiné à un fauteuil roulant électrique, il était capable de conduire, de faire de la plongée, de cuisiner à la perfection, de garder 5 de ses petits-enfants après l'école et de vivre de manière autonome, avec une aide minime de préposées pour faire sa toilette et s'habiller le matin.

Barry ne se plaint jamais de la douleur, mais quand on s'informe, il confirme qu'elle est toujours présente à différents degrés. Il a aussi des spasmes et des crampes musculaires, une sensibilité au froid et un œdème aux membres inférieurs.

Barry compose avec sa mobilité réduite et sa crainte des chutes en observant des étapes strictes pour se lever, s'asseoir dans son fauteuil roulant ou en sortir, s'habiller et préparer ses repas. Depuis 4 ans, j'ai réussi à le convaincre de porter des bas de contention et d'utiliser des bottes à compression intermittente lorsqu'il est assis. Ces bottes ont peut-être accru sa dépendance vis-à-vis d'autrui pour enfiler et retirer ses bas, mais elles ont fait diminuer son œdème et son inconfort aux membres inférieurs.

La massothérapie a contribué à soulager ses crampes musculaires aux cuisses jusqu'à ce qu'une ergothérapeute conçoive des appuis latéraux au bout du siège de son fauteuil roulant pour corriger des problèmes d'adduction.

Barry analyse continuellement ses symptômes et travaille de manière proactive à trouver les meilleures solutions possibles. Certaines de ses difficultés ont graduellement disparu. Depuis 6 mois, il n'a cependant plus confiance en sa capacité de conduire, car le temps de réaction de sa jambe et de son pied gauches dominants a ralenti. Le syndrome postpolio accentue peut-être le ralentissement normal des réactions dont nous faisons tous l'expérience en vieillissant.

Je fais de mon mieux pour aider Barry à rester autonome. Même si je suis une professionnelle de la santé à la retraite, il a fallu que je sois personnellement concernée pour me rendre compte que le personnel des aéroports, des boutiques, des hôtels, etc., a tendance à s'adresser à moi plutôt qu'à Barry dans son fauteuil roulant. Je remarque aussi que la plupart des gens ne pensent jamais aux problèmes d'accessibilité tant qu'eux-mêmes ou un proche ne sont pas personnellement touchés. - Joan M. Fisher

\section{Point de vue de la physiatre}

J'ai rencontré Barry en 2001, après le départ à la retraite de son physiatre précédent. Depuis des décennies, il était suivi dans un centre de réadaptation neuromusculaire pour son syndrome postpolio. Nous axons les consultations à la clinique autour de plusieurs objectifs, notamment le confort du fauteuil roulant, la sécurité des transferts, la conservation de l'énergie et le traitement de la douleur ${ }^{6}$. Barry se présente à tous ses rendez-vous prêt à faire le point et à poser ses questions sur les problèmes qu'il a relevés. En général, cela est suivi rapidement d'un plan dont il souhaite discuter. Par exemple, lors d'une visite, Barry a expliqué qu'après un épisode de 
maladie, il avait eu de la difficulté avec ses transferts. Je l'ai interrogé sur d'autres problèmes fonctionnels et j'ai effectué un examen neuromusculosquelettique ciblé, après quoi nous avons étudié diverses options, comme l'installation d'une barre d'appui SuperPole pour l'aider à se mettre au lit et à en sortir. Barry a d'abord dit : « Je ne crois pas être rendu là. » Et j'ai respecté son point de vue. Il a fini par accepter de faire l'essai de certains dispositifs d'aide. Ceuxci ont amélioré son fonctionnement et sa qualité de vie.

La gestion de la douleur est un enjeu constant, surtout parce que Barry avance en âge. J'ai cherché à explorer des stratégies non médicales. Au fil des ans, nous avons essayé diverses modalités, des programmes d'étirements, l'hydrothérapie et la massothérapie. Nous avons vérifié son fauteuil roulant électrique pour assurer un maximum de confort. Barry a tenu un carnet de ses activités et fait des liens avec sa perception de la douleur pour tenter de cerner les facteurs contributifs. Nous avons aussi passé en revue la conservation de l'énergie et les stratégies d'étalement des activités. À un moment, Barry s'est demandé s'il pouvait essayer la marijuana à des fins médicales. Une recension de la littérature a toutefois généré des conseils anecdotiques, d'une utilité limitée pour les personnes atteintes du syndrome postpolio. Je lui ai suggéré de travailler avec un spécialiste de la marijuana thérapeutique, et nous avons discuté des paramètres spécifiques qui permettraient d'en mesurer les effets. Il y travaille actuellement.

Barry a commencé à faire de la plongée avec un instructeur spécialisé pour les personnes handicapées. Comme il ne présentait aucune contre-indication médicale, Barry a littéralement plongé, c'est le cas de le dire. Il a constaté une réduction de sa douleur, surtout les jours où il plongeait. Nous avons exploré les facteurs qui pouvaient contribuer à ce phénomène, par exemple le fait de se divertir, l'engouement pour une activité nouvelle et stimulante, le mouvement en quasi-apesanteur et l'amélioration de la forme physique. Malheureusement, la pandémie de COVID19 a mis cette activité essentielle sur pause.

Parmi les autres difficultés rencontrées, mentionnons l'accès au financement pour l'équipement et les traitements. Les services offerts par les cliniques externes et communautaires spécialisées sont toujours à la merci des coupes budgétaires provinciales. Par exemple, il y a une pénurie de psychologues cliniciens pour aider les patients non hospitalisés qui peinent à s'adapter aux phases aiguë, chronique et progressive de leur handicap. Souvent, Barry a payé de sa poche pour les dispositifs et les services dont il avait besoin. La pandémie a exacerbé ces difficultés, prolongeant notamment les temps d'attente pour des services à domicile essentiels, comme la visite des préposées. - Sue Dojeiji

\section{Point de vue du médecin de famille}

J'ai rencontré Barry il y a 10 ans, lorsque sa femme (aujourd'hui décédée) et lui sont devenus mes patients. Il était déjà suivi en physiatrie. J'ai donc considéré que c'était une occasion de m'impliquer en tant que personne-ressource et clinicien qualifié. J'ai pu mesurer l'impact de son syndrome postpolio. Sa panoplie de symptômes - douleur, faiblesse, fatigue et autres - s'est complexifiée avec l'âge, et les ressources et les soutiens sont limités. Heureusement, et c'est tout à son honneur, Barry a une volonté de fer et il a une façon holistique de voir à sa santé; il a également un bon réseau social et une équipe soignante dévouée.

Nous travaillons ensemble à trouver des solutions; nous en avons envisagé, essayé et éliminé plusieurs. Par exemple, à un moment, nous avons songé à utiliser la marijuana à des fins médicales pour sa douleur. J'ai exprimé mon opinion à Barry au sujet des données disponibles (ou de leur absence). Je crois que c'est la qualité de notre rapport patient-médecin au fil des ans qui nous a permis d'explorer même des approches non traditionnelles.

J'ai rencontré la famille de Barry. Il est primordial que je sois au courant de l'implication de ses proches, de ses amis et de son équipe soignante dans le contexte de sa maladie et de ses soins. Je veux être prêt à collaborer avec eux en cas de problème. Avec Barry, ils sont souvent aux premières loges pour noter la moindre détérioration ou tout nouveau besoin. Barry est très près de ses filles et ensemble, ils discutent ouvertement de tout ce qui concerne son bien-être. Ses filles ont suggéré des tests; par exemple, elles ont déjà demandé un test pour la maladie cœliaque, parce que l'une d'entre elles en est atteinte. Elles se parlent s'il y a un changement dans son état de santé général et elles ont veillé à ce qu'il nomme un mandataire pour ses soins de santé.

À mesure que Barry avance en âge, j'espère demeurer l'une des personnes qui défendront ses intérêts en matière de santé afin qu'il reçoive les soins requis et, surtout, le soutien communautaire et les interventions médicales appropriées. - Rene A. Leiva

\section{Références}

1. Farbu E, Gilhus NE, Barnes MP, et al. EFNS guideline on diagnosis and management of post-polio syndrome. Report of an EFNS task force. Eur J Neurol 2006;13:795-801.

2. Connell BR, Jones M, Mace R, et al. 7 guiding principles. Dublin: Universal Design Network of Canada; 2020. Accessible ici : https://universaldesign.ie/ What-is-Universal-Design/The-7-Principles/ (consulté le 27 août 2021).

3. Koopman FS, Beelen A, Gilhus NE, et al. Treatment for post-polio syndrome. Cochrane Database Syst Rev 2015;(5):CD007818.

4. Blockmans $D$, Persoons $P$, Van Hoodenhove $B$, et al. Does methylphenidate reduce the symptoms of chronic fatigue syndrome? Am J Med 2006;119:167.e23-30.

5. Bruno R. The Type A polio survivor. International Centre for Polio Education; 2018. Accessible ici : type_a_polio_survivors.pdf (consulté le 21 oct. 2021).

6. What is a Physiatrist? Kingston (ON): Canadian Association of Physical Medicine and Rehabilitation; 2018. Accessible ici : https://www.capmr.ca/about-capmr/ what-is-a-physiatrist (consulté le 27 août 2021).

\section{Intérêts concurrents : Aucun déclaré.}

Cet article a été révisé par des pairs.

Affiliations : Patient (McMahon), Ottawa, Ont.; Centre de réadaptation de l'Hôpital d'Ottawa (Dojeiji), Ottawa, Ont.; Soins continus Bruyère (Leiva); Département de médecine familiale (Dojeiji, Leiva), Université d'Ottawa, Ottawa, Ont.; Amie (Fisher), Ottawa, Ont.

Propriété intellectuelle du contenu : Il s'agit d'un article en libre accès distribué conformément aux modalités de la licence Creative Commons Attribution (CC BY-NC-ND 4.0), qui permet l'utilisation, la diffusion et la reproduction de tout médium à la condition que la publication originale soit adéquatement citée, que l'utilisation se fasse à des fins non commerciales (c.-à-d., recherche ou éducation) et qu'aucune modification ni adaptation n'y soit apportée. Voir : https://creativecommons.org/licenses/by-nc-nd/4.0/deed.fr.

Correspondance : cmajgroup@cmaj.ca 